\title{
SR17018 stimulates atypical $\mu$-opioid receptor phosphorylation and dephosphorylation
}

\author{
Sebastian Fritzwanker ${ }^{1}$, Stefan Schulz ${ }^{1}$, and Andrea Kliewer ${ }^{1}$ \\ ${ }^{1}$ Department of Pharmacology and Toxicology, Jena University Hospital - Friedrich Schiller \\ University Jena
}

January 10, 2021

\begin{abstract}
Abstract Background and Purpose Opioid-associated overdoses and deaths due to respiratory depression are a major public health problem in the US and other western countries. During the last decade much research effort has been directed towards the development of $\mathrm{G}$ protein-biased $\mu$-opioid receptor (MOP) agonists as a possible means to circumvent this problem. The bias hypothesis proposes that $\mathrm{G}$ protein signalling mediates analgesia whereas $\beta$-arrestin signalling mediates respiratory depression. SR-17018 was initially reported as a highly biased $\mu$-opioid with an extremely wide therapeutic window. Later it was shown that SR-17018 can also reverse morphine tolerance and prevent withdrawal by a hitherto unknown mechanism of action. Experimental Approach Here, we examined the temporal dynamics of SR-17018-induced MOP phosphorylation and dephosphorylation. Key Results Exposure of MOP to saturating concentrations of SR-17018 for extended periods of time stimulated a MOP phosphorylation pattern that was indistinguishable from that induced by the full agonist DAMGO. Unlike DAMGO-induced MOP phosphorylation, which is reversible within minutes after agonist washout, SR-17018-induced MOP phosphorylation persisted for hours under otherwise identical conditions. Such a delayed MOP dephosphorylation kinetics was also found for the partial agonist buprenorphine. However unlike our observations for buprenorphine, SR-17018-induced MOP phosphorylation was fully reversible when naloxone was included in the washout solution. Conclusion and Implications SR-17018 exhibits a qualitative and temporal MOP phosphorylation profile that is strikingly different from any other known biased, partial or full MOP agonist. We conclude that detailed phosphorylation analysis may provide novel insights into previously unappreciated pharmacological properties of newly synthesized MOP ligands.
\end{abstract}

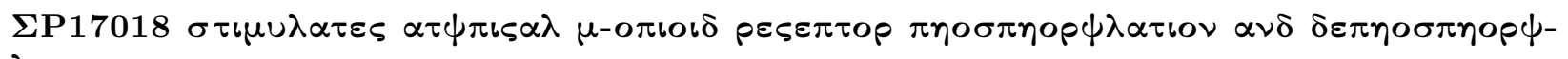
$\lambda \alpha \tau \iota \mathrm{\nu}$

Sebastian Fritzwanker ${ }^{1}$, Stefan Schulz $^{1^{*}}$ and Andrea Kliewer ${ }^{{ }^{*}}$

${ }^{1}$ Department of Pharmacology and Toxicology, Jena University Hospital - Friedrich Schiller University Jena, Drackendorfer Straße 1, D-07747 Jena, Germany

Running head: SR17018 is an atypical opioid

*Author for correspondence: Andrea Kliewer and Stefan Schulz, Institute of Pharmacology and Toxicology, Jena University Hospital - Friedrich Schiller University Jena, Drackendorfer Straße 1, D-07747 Jena, Germany, Phone: +49-3641-9325671/50, FAX: +49-3641-9325652, Email:andrea.kliewer@med.uni-jena.de and Stefan.schulz@med.uni.jena.de

Financial disclosures: S.S. is founder and shareholder of 7TM Antibodies GmbH, Jena, Germany. Alle other authors have nothing to disclose.

Statement of Conflicts of Interest: The authors have nothing to disclose 


\title{
Abbreviations:
}

GPCR G protein-coupled receptor

MOP $\mu$-opioid receptor

HEK293 human embryonic kidney 293 cells

GRK G protein-coupled receptor kinase

Key words

$\mu$-opioid receptor; DAMGO; SR-17018, buprenorphine

\section{Bullet point summary}

What is already known

SR-17018 was initially reported as a highly biased $\mu$-opioid with an extremely wide therapeutic window, that does not produce tolerance, reverse morphine tolerance and prevent withdrawal.

Recently it was shown that SR-17018 consistently exhibited low intrinsic efficacy in vitro and in vivo and showed no statistically significant bias towards or away from any G protein activation

What this study adds

SR-17018 exhibits a qualitative and temporal MOP phosphorylation profile that is strikingly different from any other known biased, partial or full MOP agonist.

Clinical significance

Due to the unusual pharmacological profile SR-17018 belongs to a new class of atypical opioids.

\begin{abstract}
Background and Purpose

Opioid-associated overdoses and deaths due to respiratory depression are a major public health problem in the US and other western countries. During the last decade much research effort has been directed towards the development of $\mathrm{G}$ protein-biased $\mu$-opioid receptor (MOP) agonists as a possible means to circumvent this problem. The bias hypothesis proposes that $\mathrm{G}$ protein signalling mediates analgesia whereas $\mathrm{B}$-arrestin signalling mediates respiratory depression. SR-17018 was initially reported as a highly biased $\mu$-opioid with an extremely wide therapeutic window. Later it was shown that SR-17018 can also reverse morphine tolerance and prevent withdrawal by a hitherto unknown mechanism of action.
\end{abstract}

Experimental Approach

Here, we examined the temporal dynamics of SR-17018-induced MOP phosphorylation and dephosphorylation.

Key Results

Exposure of MOP to saturating concentrations of SR-17018 for extended periods of time stimulated a MOP phosphorylation pattern that was indistinguishable from that induced by the full agonist DAMGO. Unlike DAMGO-induced MOP phosphorylation, which is reversible within minutes after agonist washout, SR-17018induced MOP phosphorylation persisted for hours under otherwise identical conditions. Such a delayed MOP dephosphorylation kinetics was also found for the partial agonist buprenorphine. However unlike our observations for buprenorphine, SR-17018-induced MOP phosphorylation was fully reversible when naloxone was included in the washout solution.

Conclusion and Implications 
SR-17018 exhibits a qualitative and temporal MOP phosphorylation profile that is strikingly different from any other known biased, partial or full MOP agonist. We conclude that detailed phosphorylation analysis may provide novel insights into previously unappreciated pharmacological properties of newly synthesized MOP ligands.

\section{Introduction}

Opioids are the most effective drugs for the treatment of severe pain. However, their clinical use in acute and chronic pain is limited by severe adverse side effects such as respiratory depression, constipation, dependence, and development of tolerance (Koob et al., 2009; Williams et al., 2013). Until now opioid-associated overdoses and deaths due to respiratory depression from prescription opioids are a major public health problem in the US and other western countries. It is believed that one way to solve this problem may be the development of biased $\mu$-opioid receptor (MOP) agonists. These compounds have been developed based on the hypothesis that selective activation of the G protein signal pathway via MOP mediates the analgesic effect by avoiding stimulation of $B$-arrestin signalling, which is believed to induce adverse opioid effects such as respiratory depression or constipation.

SR-17018 is one of the most recent described G protein-biased agonists (Schmid et al., 2017; Gillis et al., 2020). Schmid et al. (2017) demonstrated an extremely high bias factor in different G protein assays over B-arrestin 2 recruitment in vitro, and significant separation between antinociception and respiratory side effects in vivo. In addition to the extremely wide therapeutic window, it was reported later that SR-17018 does not produce tolerance in the hot plate antinociception assay (Grim et al., 2020). Furthermore, it was shown that SR-17018 can reverse morphine tolerance and prevent withdrawal by an unknown mechanism of action (Grim et al., 2020).

In contrast, a more recent study by Gillis et al. (2020) showed that SR-17018 consistently exhibited low intrinsic efficacy across a variety of assays and showed no statistically significant bias towards or away from any G protein activation. Furthermore, similar kinetics were observed between antinociception and respiratory depressant effects. Similar in vitro results were obtained with the partial agonist buprenorphine, however with an increased therapeutic window regarding respiratory depression (Gillis et al., 2020; McPherson et al., 2010; Pergolizzi et al., 2010). In clinical settings buprenorphine is used as an alternative to methadone in the treatment of heroin addiction, due to its mixed agonist-antagonist properties (Lufty et al., 2003). Collectively, these findings suggest that SR-17018 may be similar to buprenorphine and exhibit partial agonistic properties.

During the last decade, we have shown that the phosphorylation barcode of MOP carboxyl-terminal tail is dependent on agonist efficacy and indicative of $B$-arrestin recruitment and receptor internalization (Just et al., 2013; Miess et al., 2018). High-efficacy MOP agonists like DAMGO and fentanyl induce a robust hierarchical and sequential multisite receptor phosphorylation, whereas low-efficacy agonist, like morphine, oxycodone and buprenorphine, trigger only phosphorylation at Ser ${ }^{375}$. Recent phosphorylation studies with SR-17018 have reported an unusual phosphorylation pattern, which is limited to Ser $^{375}$ during the first 20 min of stimulation, corresponding to a low-efficacy agonist (Gillis et al., 2020). In contrast, incubation for $>30$ min leads to a multisite receptor phosphorylation, which corresponds to a high-efficacy agonist. Furthermore, SR-17018 induced MOP phosphorylation is driven by GRK2/3 and is naloxone sensitive.

Given its unusual pharmacological profile and unknown mechanism of action, we performed a series of MOP phosphorylation and dephosphorylation experiments in vitro and compared the effects of SR-17018 with the partial agonist buprenorphine.

\section{Material}

\section{Reagents and antibodies}

$\left[\mathrm{D}-\mathrm{Ala}^{2}, \mathrm{~N}-\mathrm{Me}-\mathrm{Phe}^{4}, \mathrm{Gly}^{5}\right.$-ol]-Enkephalin acetate salt (DAMGO) was purchased from Sigma Aldrich (Munich, Germany). SR-17018 was obtained from MedChemExpress (Monmouth Junction, USA), buprenorphine from Indivior (Dublin, Ireland) and naloxone from ratiopharm (Ulm, Germany). Pierce ${ }^{\mathrm{TM}} \mathrm{HA}$ epitope 
tag antibodies were obtained from Thermo Scientific (Rockford, USA). The rabbit polyclonal phosphositespecific $\mu$-opioid receptor antibodies anti-pT370 $\{3195\}$, anti-pT376 $\{3723\}$, anti-pT379 \{3686\} and antipS375 $\{5375\}$ were generated and extensively characterized as previously described (\{numbers $\}$ indicate internal inventory numbers) (Doll et al., 2012; Just et al., 2013; Yousuf et al., 2015). Generation, characterization and use of the polyclonal rabbit anti-HA antibody $\{0631\}$ was described previously (Pfeiffer et al., 2002). The secondary HRP-linked anti-rabbit antibody was purchased from Cell Signaling (Frankfurt, Germany)

\section{Methods}

\section{Cell culture and transfection}

HEK293 cells were originally obtained from the German Resource Centre for Biological Material (DSMZ, Braunschweig, Germany) and grown in Dulbecco's modified Eagle's medium supplemented with 10\% fetal calf serum, $2 \mathrm{mM} \mathrm{L}$-glutamine and $100 \mathrm{U} / \mathrm{ml}$ penicillin/streptomycin and cultured in a humidified atmosphere containing $5 \% \mathrm{CO}_{2}$. Cells were stable transfected with mouse MOP-HA.

\section{Western Blot assay}

HEK293 cells stably expressing the HA-MOP were seeded onto poly-L-lysine-coated $60 \mathrm{~mm}$ dishes and grown to $90 \%$ confluency. After agonist-stimulation, cells were lysed in RIPA buffer (50 mM Tris-HCl, pH 7.4, $150 \mathrm{mM} \mathrm{NaCl}, 5 \mathrm{mM}$ EDTA, $1 \%$ Nonidet P-40, 0,5\% sodium deoxycholate, 0,1 \% SDS) containing protease and phosphatase inhibitors (Complete mini and PhosSTOP; Roche Diagnostics, Mannheim, Germany). When indicated, cells were washed three times with either PBS (washout) or PBS supplemented with $10 \mu \mathrm{M}$ naloxone (naloxone washout) containing $90 \mathrm{mM} \mathrm{NaCl}$ and $50 \mathrm{mM}$ sodium citrate (pH 4.5). After removal of agonist, the cells were incubated in the absence of agonist at $37^{\circ} \mathrm{C}$ as indicated and lysed in RIPA buffer containing protease and phosphatase inhibitors, as described before. Pierce ${ }^{\mathrm{TM}} \mathrm{HA}$ epitope tag antibody beads (Thermo Scientific, Rockford, USA) were used to enrich HA-tagged MOP. To elute proteins from the beads, the samples were incubated in SDS sample buffer for $25 \mathrm{~min}$ at $43^{\circ} \mathrm{C}$. Supernatants were separated from the beads, loaded on $8 \%$ SDS polyacrylamide gels and immunoblotted onto nitrocellulose membranes afterwards. After blocking, membranes were incubated with anti-pT370, anti-pS375, anti-pT376 or anti- pT379 antibody overnight. Membranes were incubated in secondary antibody for 2 hours, followed by detection using a chemiluminescence system (90 mM p-coumaric-acid, $250 \mathrm{mM}$ luminol, $30 \%$ hydrogen peroxide). Blots were subsequently stripped and incubated again with the phosphorylation-independent anti-HA antibody to confirm equal loading of the gels. Protein bands on Western blots were exposed to X-ray films. Western blotting protocols comply with the BJP recommendations for immunoblotting (Alexander et al., 2018).

\section{Immunocytochemistry}

HEK-293 HA-MOP cells were seeded onto poly-L-lysine coated 24-well plates overnight. On the next day, cells were pre-incubated with anti-HA antibody for $2 \mathrm{~h}$ at $4^{\circ} \mathrm{C}$. Cells were then transferred to $37^{\circ} \mathrm{C}$, exposed to $10 \mu \mathrm{M}$ agonist for $30 \mathrm{~min}$ at $37^{\circ} \mathrm{C}$ and fixed with $4 \%$ paraformaldehyde and $0.2 \%$ picric acid in phosphate buffer ( $\mathrm{pH}$ 6.9) for 30 minutes at room temperature. After washing the coverslips with PBS w/o Ca2+/Mg2+ buffer several times, cells were blocked with phosphate buffer containing 3\% NGS for 2 hours and were then incubated with Alexa488-conjugated secondary antibody (1:2,000) (LifeTechnologies, Thermo Fisher Scientific A11008). Specimens were mounted with Roti@-MountFluorCare DAPI (Carl Roth, HP20.1) and examined using a Zeiss LSM510 META laser scanning confocal microscope. Immunocytochemistry protocols comply with the BJP recommendations for immunocytochemistry (Alexander et al., 2018).

\section{Data Availability}

The authors declare that all data supporting the findings of this study are presented within the paper and its supporting information files. The data that support the findings of this study are available from the authors upon reasonable request.

\section{Results}


SR-17018 was developed as a G protein-biased MOP agonist but exhibits a number of pharmacological effects, which cannot be explained by the biased signaling hypothesis. To better understand SR-17018 ligand properties, we performed a series of MOP phosphorylation and dephosphorylation experiments comparing SR-17018 to the low-efficacy agonist buprenorphine and the full agonist DAMGO as internal standard.

First, we evaluated dose- and time-dependent MOP phosphorylation induced by SR-17018 or buprenorphine. As shown in Figure 1, 30 min-exposure to saturating concentrations of SR-17018 induces a multi-site phosphorylation that was indistinguishable from that induced by DAMGO. In contrast, buprenorphine induces only a robust $\mathrm{Ser}^{375}$ phosphorylation under otherwise identical conditions. As depicted in Figure 2, DAMGO stimulated MOP phosphorylation occurred rapidly within seconds to minutes. In contrast, both SR-17018 and buprenorphine-mediated MOP phosphorylation required extended exposure times ( $>20 \mathrm{~min})$. Furthermore, SR-17018 and DAMGO promote a robust internalization whereas buprenorphine failed to stimulated any detectable MOP endocytosis.

Next, we evaluated the temporal dynamics of MOP dephosphorylation after extensive ligand washout with PBS (Figure 3). While DAMGO-induced phosphorylation was quickly reversed within 5 to 10 min after agonist removal, SR-17018-mediated MOP phosphorylation was retained for hours under otherwise identical conditions. Similar, the buprenorphine-stimulated Ser ${ }^{375}$ phosphorylation was also resistant to PBS washout (Figure 3). Interestingly, when $10 \mu \mathrm{M}$ naloxone were included into the washout solution, MOP dephosphorylation was strongly facilitated in SR-17018-treated but not in buprenorphine-treated cultures (Figure 4).

\section{Discussion}

SR-17018 is unique in that it exhibits an atypical MOP phosphorylation and dephosphorylation profile (Gillis et al., 2020). Saturating concentrations of SR-17018 stimulate a full agonist-like multisite phosphorylation of MOP but with delayed onset ( $>20 \mathrm{~min})$. A similar slow phosphorylation kinetics is observed with the partial agonists buprenorphine, whereas the full agonist DAMGO induces full MOP phosphorylation within seconds. At least three kinases contribute to agonist-induced MOP phosphorylation namely GRK2, GRK3 and GRK5 (Just et al., 2013; Doll et al., 2012). The most likely explanation is that SR-17018, buprenorphine and DAMGO restrain the receptor in different conformations, which exhibit different affinities for individual GRKs (Gillis et al., 2020).

Conversely, dephosphorylation of DAMGO-activated MOPs occurs within minutes after agonist washout. In contrast, SR-17018-stimulated MOP phosphorylation occurs in a delayed manner similar to that observed with buprenorphine and persisted for hours after agonist washout. These data suggest that SR-17018 remains tightly bound to the MOP receptor after washout, while preventing receptor dephosphorylation. However, SR-17018-induced MOP phosphorylation was reversible when the antagonist naloxone was included in aqueous washout solutions. In contrast, buprenorphine-stimulated MOP phosphorylation was not reversible by naloxone. These results predict that SR-17018 has a very slow off-rate at MOP similar to that known for buprenorphine (Gillis et al., 2020; Pedersen et al., 2020). However, SR-17018 has a much lower affinity than buprenorphine so that it can easily be displaced by naloxone (Pedersen et al., 2020; Schmid et al., 2017).

SR-17018 exhibits a peculiar pharmacological profile in preclinical animals models where it was shown to prevent opioid withdrawal signs (Schmid et al., 2017; Grim et al., 2020). Such activity has previously been observed for buprenorphine but not with any other biased MOP agonist (Lutfy et al., 2013; Fishman \& Kim, 2018). This suggests that opioids with delayed dephosphorylation kinetics may be useful for opioid maintenance therapy. Nevertheless, SR-17018 differs from buprenorphine in that its effects are easily reversible with naloxone.

Together, the present study reveals a mechanism of action for SR-17018 that is clearly different from any other known MOP agonist. Our findings also demonstrate that newly synthesized compounds should be fully characterized including detailed analysis of their receptor phosphorylation kinetics before classification as biased, partial or full agonists. 


\section{End notes}

Supplementary Information is available in the online version of the paper

Acknowledgements We thank Heike Stadtler for excellent technical assistance and Dr. Rainer K. Reinscheid for critical reading of the manuscript. This work was supported by the Else Kröner Fresenius Stiftung (2019_A68), Interdisciplinary Center for Clinical Research Jena (AMSP 03) to A.K and Deutsche Forschungsgemeinschaft grants SFB/TR166-TPC5, SCHU924/15-1 and SCHU924/18-1 to S.S.

Author contribution A.K. and S.S. initiated the project and designed all experiments with S.F.. S.F. performed all in vitro studies. The manuscript was written by A.K., S.S. and S.F.

\section{References}

Alexander SPH, Roberts RE, Broughton BRS, Sobey CG, George CH, Stanford SC, et al. (2018). Goals and practicalities of immunoblotting and immunohistochemistry: A guide for submission to the British Journal of Pharmacology. Br J Pharmacol 175: 407-411.

Doll C, Poll F, Peuker K, Loktev A, Gluck L, \& Schulz S (2012). Deciphering micro-opioid receptor phosphorylation and dephosphorylation in HEK293 cells. Br J Pharmacol 167: 1259-1270.

Fishman MA, \& Kim PS (2018). Buprenorphine for Chronic Pain: a Systemic Review. Curr Pain Headache Rep 22: 83.

Gillis A, Gondin AB, Kliewer A, Sanchez J, Lim HD, Alamein C, et al. (2020). Low intrinsic efficacy for G protein activation can explain the improved side effect profiles of new opioid agonists. Sci Signal 13.

Grim TW, Schmid CL, Stahl EL, Pantouli F, Ho JH, Acevedo-Canabal A, et al. (2020). A G protein signaling-biased agonist at the mu-opioid receptor reverses morphine tolerance while preventing morphine withdrawal. Neuropsychopharmacology 45: 416-425.

Just S, Illing S, Trester-Zedlitz M, Lau EK, Kotowski SJ, Miess E, et al. (2013). Differentiation of opioid drug effects by hierarchical multi-site phosphorylation. Mol Pharmacol 83: 633-639.

Koob GF (2009). Neurobiological substrates for the dark side of compulsivity in addiction. Neuropharmacology 56 Suppl 1: 18-31.

Lutfy K, Eitan S, Bryant CD, Yang YC, Saliminejad N, Walwyn W, et al. (2003). Buprenorphine-induced antinociception is mediated by mu-opioid receptors and compromised by concomitant activation of opioid receptor-like receptors. J Neurosci 23: 10331-10337.

McPherson J, Rivero G, Baptist M, Llorente J, Al-Sabah S, Krasel C, et al. (2010). mu-opioid receptors: correlation of agonist efficacy for signalling with ability to activate internalization. Mol Pharmacol 78: 756766.

Miess E, Gondin AB, Yousuf A, Steinborn R, Mosslein N, Yang Y, et al. (2018). Multisite phosphorylation is required for sustained interaction with GRKs and arrestins during rapid mu-opioid receptor desensitization. Sci Signal 11.

Pedersen MF, Wrobel TM, Marcher-Rorsted E, Pedersen DS, Moller TC, Gabriele F, et al. (2020). Biased agonism of clinically approved mu-opioid receptor agonists and TRV130 is not controlled by binding and signaling kinetics. Neuropharmacology 166: 107718.

Pergolizzi J, Aloisi AM, Dahan A, Filitz J, Langford R, Likar R, et al. (2010). Current knowledge of buprenorphine and its unique pharmacological profile. Pain Pract 10: 428-450.

Pfeiffer M, Koch T, Schroder H, Laugsch M, Hollt V, \& Schulz S (2002). Heterodimerization of somatostatin and opioid receptors cross-modulates phosphorylation, internalization, and desensitization. J Biol Chem 277: 19762-19772. 
Schmid CL, Kennedy NM, Ross NC, Lovell KM, Yue Z, Morgenweck J, et al. (2017). Bias Factor and Therapeutic Window Correlate to Predict Safer Opioid Analgesics. Cell 171: 1165-1175 e1113.

Williams JT, Ingram SL, Henderson G, Chavkin C, von Zastrow M, Schulz S, et al. (2013). Regulation of mu-opioid receptors: desensitization, phosphorylation, internalization, and tolerance. Pharmacol Rev 65: 223-254.

Yousuf A, Miess E, Sianati S, Du YP, Schulz S, \& Christie MJ (2015). Role of Phosphorylation Sites in Desensitization of micro-Opioid Receptor. Mol Pharmacol 88: 825-835.

\section{Figure Legends}

Figure 1 Dose-dependent multi-site phosphorylation of SR-17018 and buprenorphine . HEK293 cells stably expressing HA-MOP were either treated with (A ) SR-17018 or (B) buprenorphine with concentrations ranging from $10 \mu \mathrm{M}$ to $1 \mathrm{nM}$ for $30 \mathrm{~min}$. Cells were lysed and immunoblotted with the anti-pT370 (pT370), anti-pT376 (pT376), anti-pT379 (pT379) or anti-pSer375 (pS375) antibody. Blots were stripped and re-probed with the phosphorylation independent anti-HA-tag antibody to confirm equal loading of the gels. Positions of molecular mass markers are indicated on the left (in $\mathrm{kDa}$ ). (A, B right lane) $10 \mu \mathrm{M}$ DAMGO samples were used as a control to visualize different development times on X-ray films. Blots are representative of three to four independent experiments.

Figure 2 Time-course of multi-site phosphorylation by DAMGO, SR-17018 and buprenorphine . (upper panel) HEK293 cells stably expressing HA-MOP were incubated with either $10 \mu \mathrm{M}$ DAMGO, SR-17018 or buprenorphine for the indicated time periods at room temperature. Cells were lysed and immunoblotted with the anti-pT370 (pT370), anti-pT376 (pT376), anti-pT379 (pT379) or anti-pSer375 (pS375) antibody. Blots were stripped and re-probed with the phosphorylation independent anti-HA-tag antibody to confirm equal loading of the gels (MOP). Positions of molecular mass markers are indicated on the left (in $\mathrm{kDa}$ ). Blots are representative of four to five independent experiments. (lower panel) HEK-293 HA-MOP cells were pre-incubated with anti-HA antibody for $2 \mathrm{~h}$ at $4^{\circ} \mathrm{C}$. Afterwards cells were treated with either $10 \mu \mathrm{M}$ DAMGO, SR-17018 or buprenorphine for $30 \mathrm{~min}$ at $37^{\circ} \mathrm{C}$. After fixation, the cells were incubated with Alexa488-conjugated secondary antibody, and examined by confocal microscopy. Shown are representative images of 3 independent experiments.

Figure 3 Time-course of multi-site dephosphorylation following stimulation with DAMGO, SR-17018 or buprenorphine.(A) HEK293 cells stably expressing HA-MOP were incubated with either $10 \mu \mathrm{M}$ DAMGO (left panels), SR-17018 (center panels) or buprenorphine (right panels) for 30 min. Cells were washed three times with PBS buffer (PBS washout), and then incubated in the absence of agonist for $0,2,5,10,20$ or 60 min at $37^{\circ} \mathrm{C}$. (B) HEK293 cells stably expressing HA-MOP were incubated with $10 \mu \mathrm{M}$ SR-17018 for $30 \mathrm{~min}$. Cells were washed three times with PBS buffer, and then incubated in the absence of agonist for $0,2,4$ or 6 hours at $37^{\circ} \mathrm{C}$. (A, B) Cells were lysed and immunoblotted with the anti-pT370 (pT370), anti-pT376 (pT376), anti-pT379 (pT379) or anti-pSer375 (pS375) antibody. Blots were stripped and re-probed with the phosphorylation independent anti-HA-tag antibody to confirm equal loading of the gels (MOP). Positions of molecular mass markers are indicated on the left (in $\mathrm{kDa}$ ). Blots are representative of three independent experiments.

Figure 4 Time-course of multi-site dephosphorylation after naloxone washout. HEK293 cells stably expressing HA-MOP were incubated with (left panel) $10 \mu \mathrm{M}$ DAMGO, (middle panel) $10 \mu \mathrm{M}$ SR-17018 or (right panel) buprenorphine for $30 \mathrm{~min}$. Cells were washed three times with $10 \mu \mathrm{M}$ naloxone and then incubated in the absence of agonist for $0,2,5,10,20$ or $60 \mathrm{~min}$ at $37^{\circ} \mathrm{C}$. Cells were lysed and immunoblotted with the anti-pT370 (pT370), anti-pT376 (pT376), anti-pT379 (pT379) or anti-pSer375 (pS375) antibody. Blots were stripped and re-probed with the phosphorylation independent anti-HA-tag antibody to confirm equal loading of the gels (MOP). Positions of molecular mass markers are indicated on the left (in $\mathrm{kDa}$ ). Blots are representative of three to five independent experiments.

Figure 1 


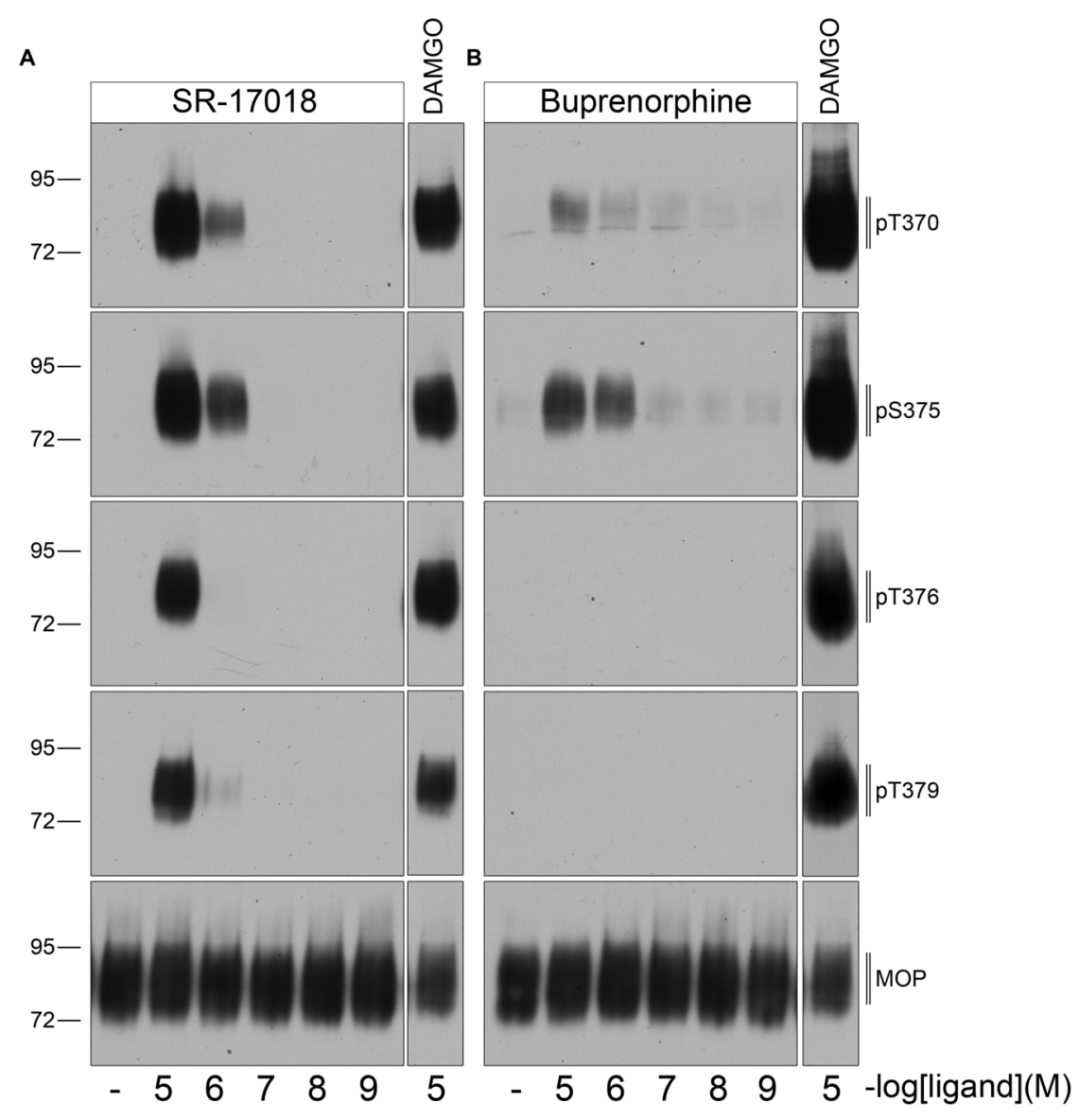

Figure 2 


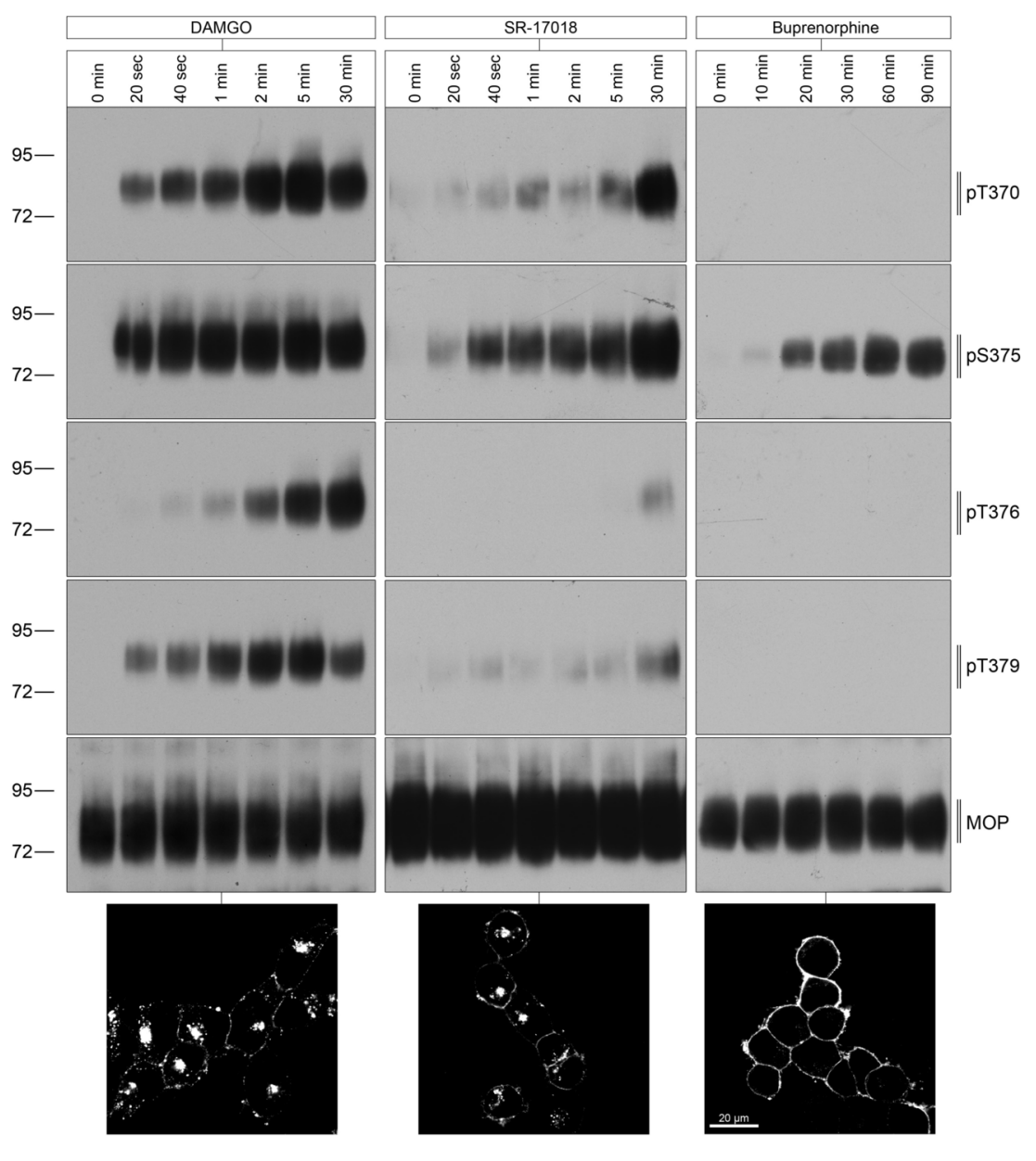

Figure 3 


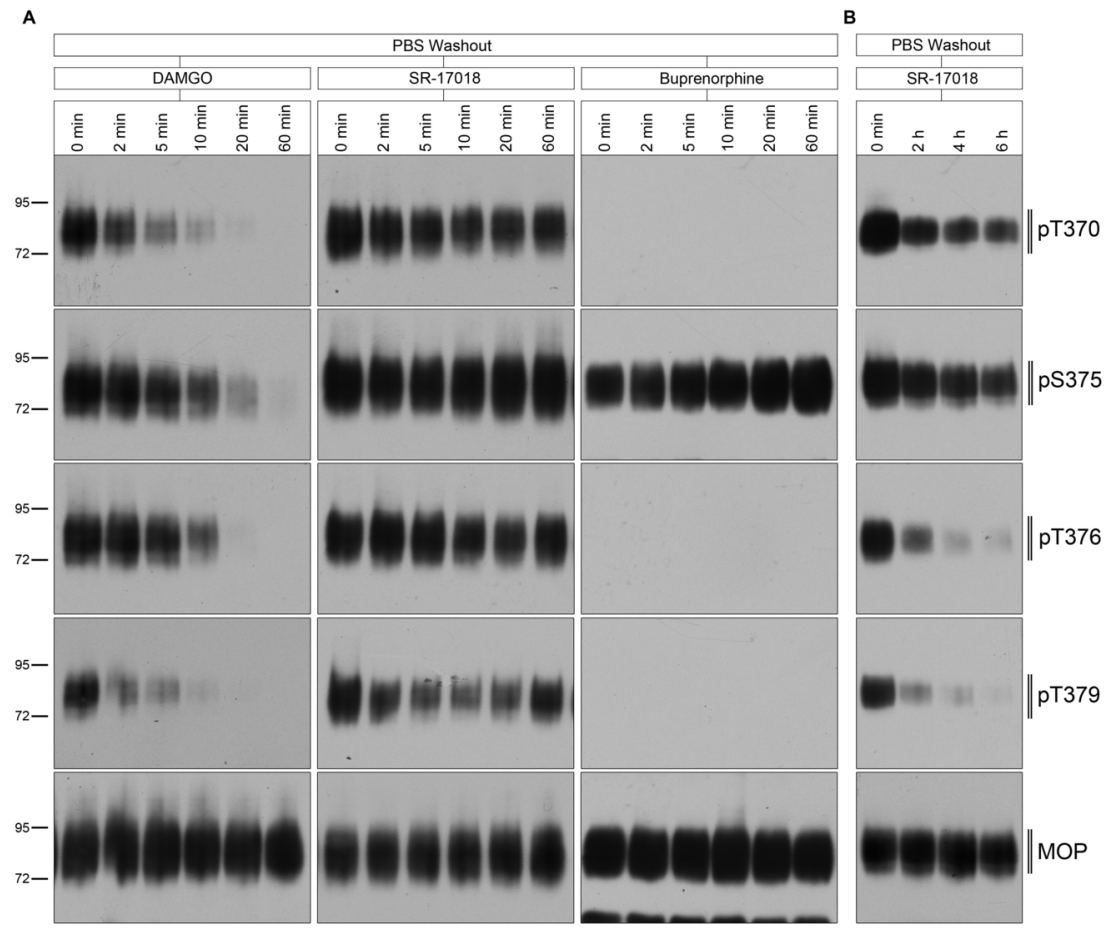

Figure 4 


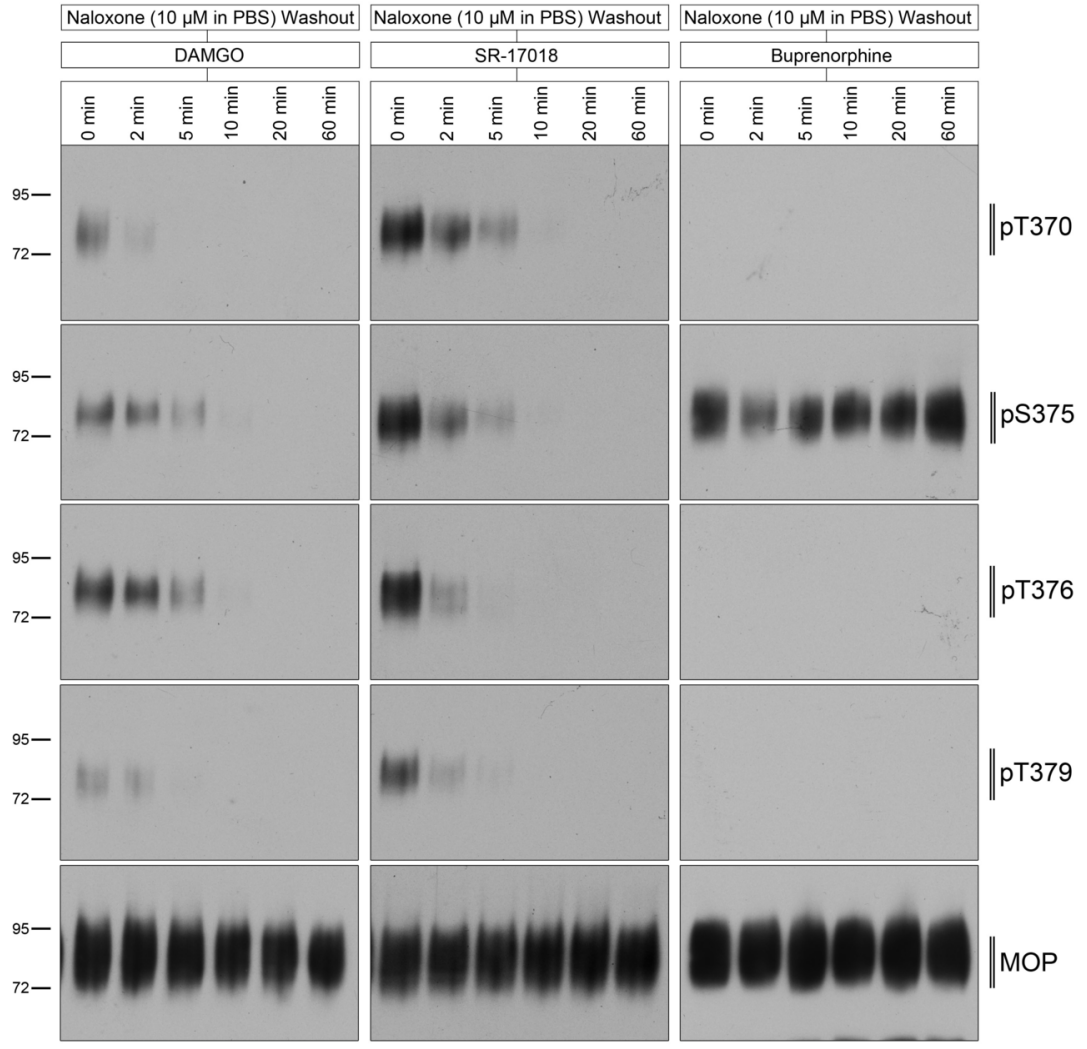




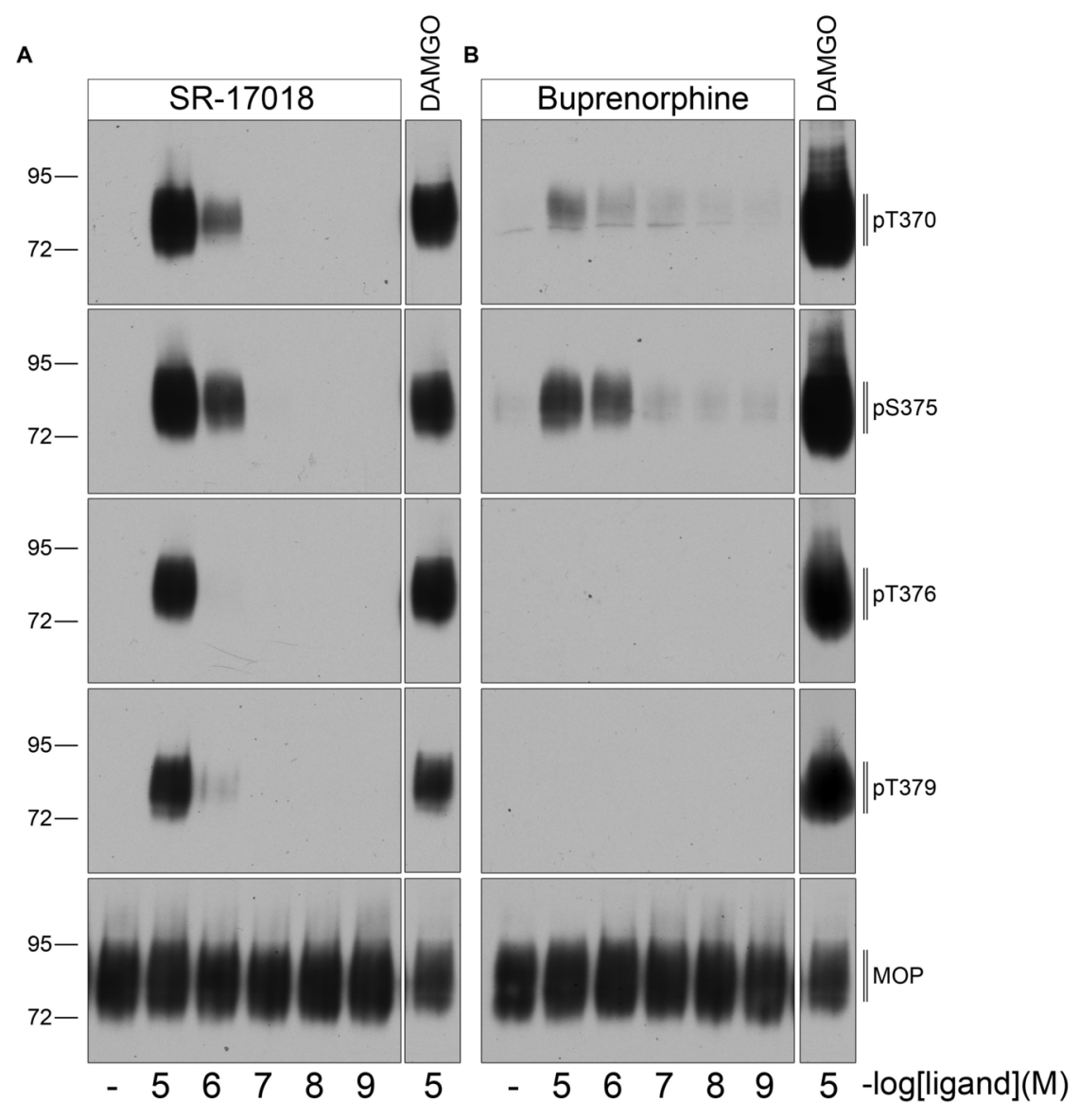




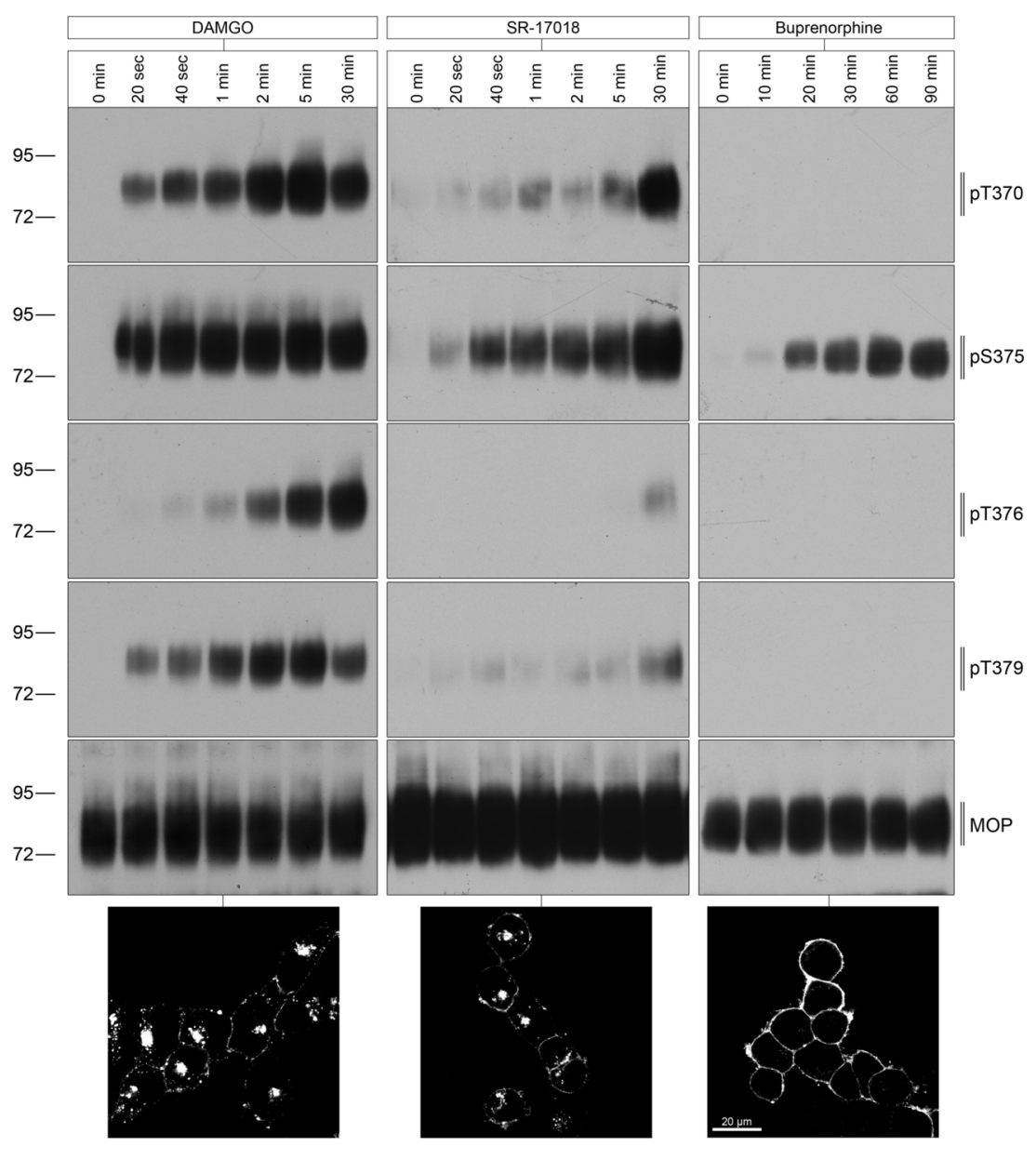


A

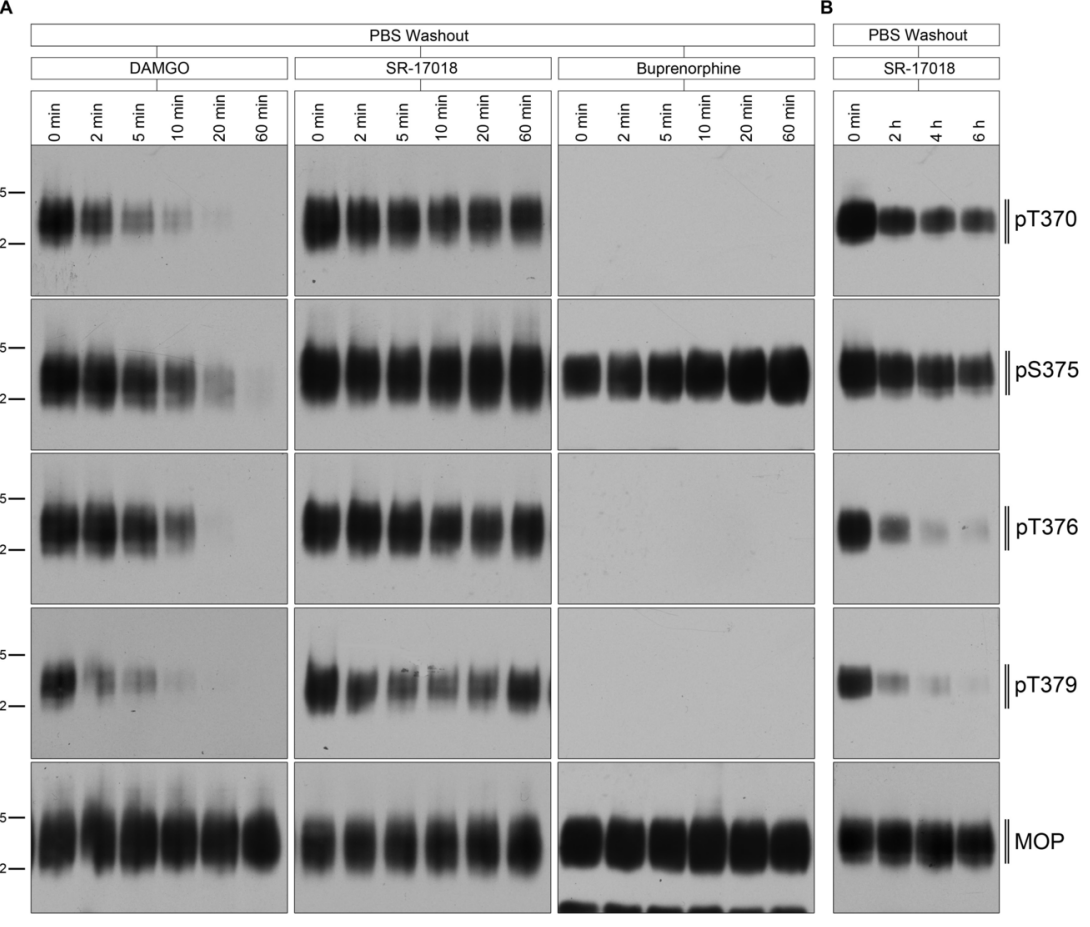

Naloxone (10 $\mu \mathrm{M}$ in PBS) Washout Naloxone (10 $\mu \mathrm{M}$ in PBS) Washout Naloxone $(10 \mu \mathrm{M}$ in PBS) Washout

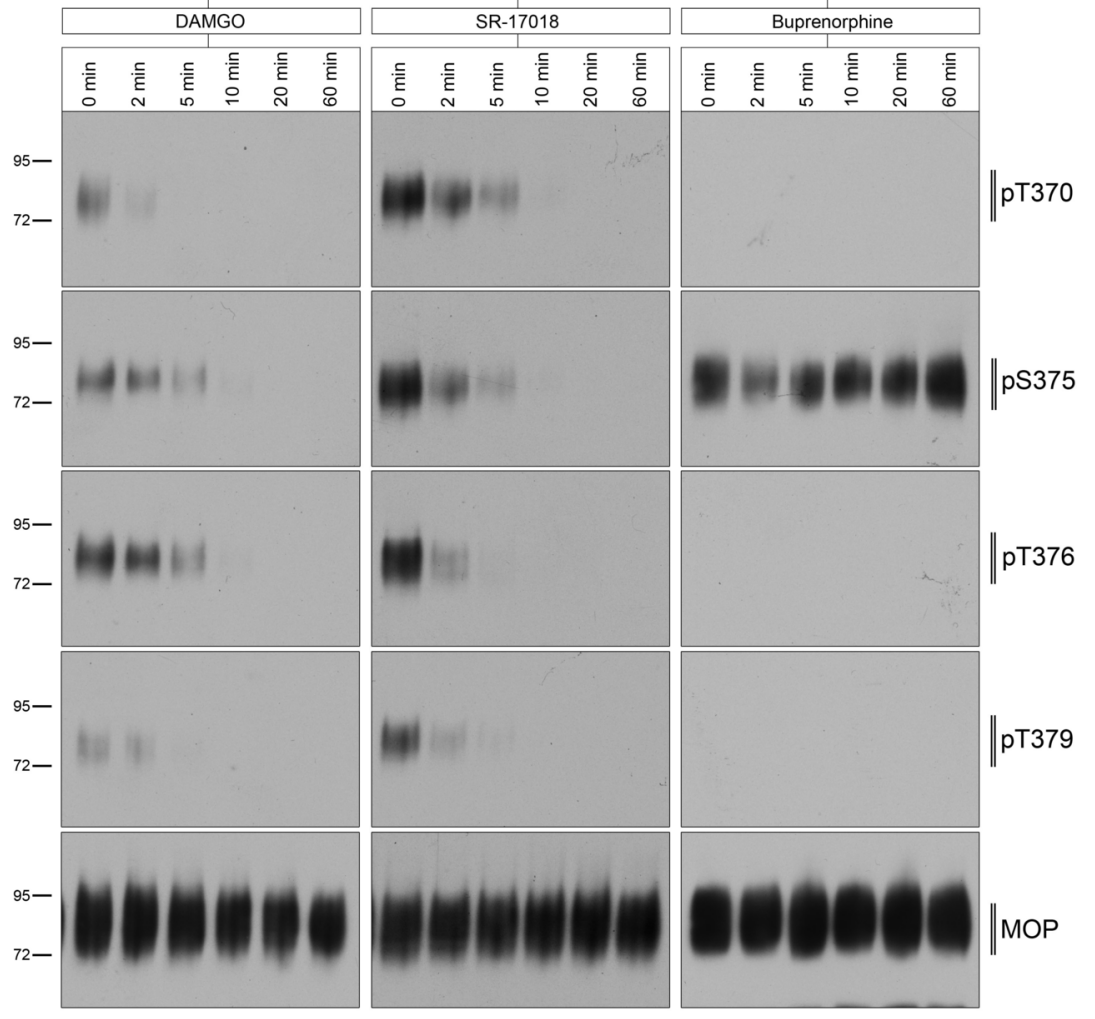

